\title{
ON THE WELL-POSEDNESS OF A MATHEMATICAL MODEL FOR LITHIUM-ION BATTERY SYSTEMS*
}

\author{
JINBIAO $\mathrm{WU}^{\dagger}$, JINCHAO XUं $\mathrm{XN}^{\ddagger}$ AND HENGHUI ZOU ${ }^{\S}$ \\ In honor of Professor Björn Engquist for his 60th birthday
}

\begin{abstract}
In this paper, we shall establish the well-posedness of a mathematical model for a special class of electrochemical power device - lithium-ion battery. The underlying partial differential equations in the model involve a (mix and fully) coupled system of quasi-linear elliptic and parabolic equations. By exploring some special structure, we are able to adopt the well-known Nash-MoserDeGiorgi boot strap to establish suitable a priori supremum estimates for the electric potentials. Using the supremum estimates, we apply the Leray-Schauder theory to establish the existence and uniqueness of a subsystem of elliptic equations that describe the electric potentials in the model. We then employ a Schauder fix point theorem to obtain the local (in time) existence for the whole model. We also consider the global existence of a modified 1-d governing system under additional assumptions. In particular, we are able to derive uniform a priori estimates depending only on the existence time $T$, including the supremum estimates for electric potentials and growth and decay estimates for the concentration $c$. Using the uniform estimates, we prove that the modified system has a solution for all time $t>0$.
\end{abstract}

Key words. Lithium-ion battery; Nash-Moser-DeGiorgi boot strap; fixed point theorems; a priori estimates; Newton-Krylov-multigrid method

AMS subject classifications. 35G25, 35G30; 35J55, 35J70; 35K15, 35K20; 35M10

1. Introduction. In this paper, we study a special class of coupled ellipticparabolic system of nonlinear partial differential equations which has its origin in the mathematical modelling of electro-chemical devices such as Lithium-ion battery system. This system of equations can be prescribed on a bounded domain $\Omega \subset \mathbf{R}^{n}$ $(1 \leq n \leq 3)$ such that

$$
\bar{\Omega}=\bar{\Omega}_{a} \cup \bar{\Omega}_{s} \cup \bar{\Omega}_{c} \subset \mathbf{R}^{n},
$$

where $\Omega_{a}, \Omega_{s}$ and $\Omega_{c}$ are rectangular subdomains of $\Omega$ that correspond to the negative electrode, the separator and the positive electrode of the Lithium ion battery respectively, see Fig.1.1 below.

Set $\Omega^{\prime}=\Omega_{a} \cup \Omega_{c}$. The system of partial differential equations that we are interested takes the following form:

$$
\begin{gathered}
-\nabla \cdot\left(\kappa(c) \nabla \Phi_{e}\right)+\nabla \cdot\left(\alpha_{1} \kappa(c) \nabla \ln c\right)-S_{e}=0, \quad x \in \Omega \\
-\nabla \cdot\left(\sigma \nabla \Phi_{s}\right)+S_{e}\left(\Phi_{s}-\Phi_{e}, c\right)=0, \quad x \in \Omega^{\prime}, \\
\frac{\partial\left(\varepsilon_{e} c\right)}{\partial t}-\nabla \cdot(D \nabla c)-S_{c}\left(\Phi_{s}-\Phi_{e}, c\right)=0, \quad x \in \Omega,
\end{gathered}
$$

*Received December 8, 2005; accepted for publication May 26, 2006.

${ }^{\dagger}$ LMAM and School of Mathematical Science, Peking University, Beijing 100871, People's Republic of China (jwu@math.pku.edu.cn). The first author was supported in part by NSFC 10501001 and SRF for ROCS, SEM.

${ }^{\ddagger}$ LMAM and School of Mathematical Science, Peking University and Department of Mathematics, Penn State University, University Park, PA 16802, USA (xu@math.psu.edu). http://www.math.psu.edu/xu/. The second author was supported in part by Changjiang Professorship through Peking University, by the Center for the Computational Mathematics and Applications, Penn State University and by NSF DMS-0209497, DMS-0308946 and NSFC-10528102.

$\S$ Department of Mathematics, University of Alabama at Birmingham, Birmingham, AL 35294 , USA (zou@math.uab.edu). 


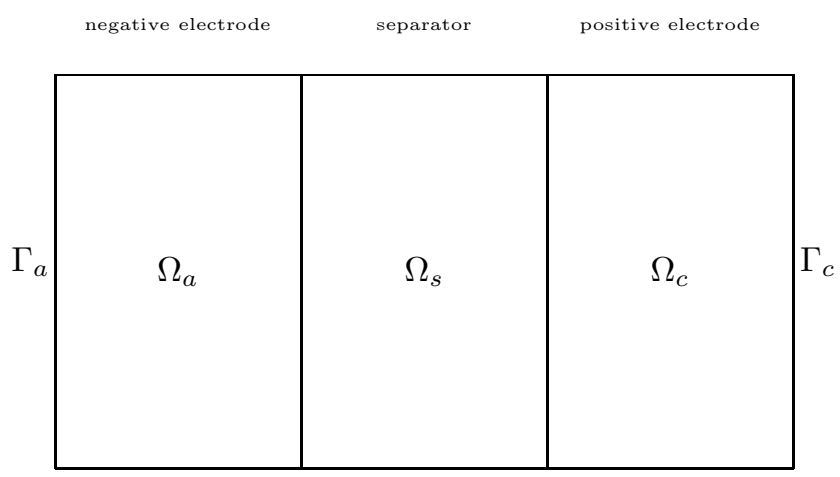

FIG. 1.1. The domain $\Omega$

Here $\Phi_{e}$ and $\Phi_{s}$ are electric potentials (in the electrolyte phase and solid phase respectively) and $c$ is the concentration of the lithium ion in the electrolyte. Proper Neumann boundary conditions are prescribed for $\Phi_{e}, \Phi_{s}$ and, together with an initial condition, for $c$.

The above system of equation represents certain mathematical model for the Lithium-ion battery system. Details of this model can be found in Gu and Wang [8], Wang [16], Fuller et al [5] and [3, 2, 9]. Similar but more complicated model equations can also be found in the modelling of other electrochemical device such as fuel cell systems, see [16].

In this paper, we are mainly interested in the new mathematical features that present in this type of partial differential equations. Equations (1.1-1.3) is a fully coupled system of quasi-linear elliptic and parabolic partial differential equations. There are at least two distinctive features that contribute to the complication of this system. First of all, the system is highly degenerate as the diffusion coefficients $\kappa(c)$ vanishes when $c=0$ and $\sigma \equiv 0$ on some entire subdomain $\Omega_{s}$ (the separator). Secondly, the "source" terms $S_{c}$ and $S_{e}$ are highly nonlinear with respect to $\Phi_{s}$ and $\Phi_{e}$ and singular at $c=0$.

To our knowledge, such coupled elliptic-parabolic partial differential equations with the aforementioned complications have not been well-studied in the literature. By exploring a special structure in the system, we are able to employ the well-known Nash-Moser-DeGiorgi boot-strap [14, 15] (which is often used for scalar equation) to establish 'conditional' a priori supremum estimates for the elliptic sub-system (1.1) and (1.2) that describe the electric potentials in the model. With these estimates, we use the Leray-Schauder theory to establish the existence and uniqueness of this sub-system. A Schauder fix point theorem is then applied (to the concentration $c$ ) to establish the local (in time) existence for the entire system (1.1-1.3).

The global (in time) existence of the solutions to the model is a more complex matter and it is not yet established in this paper. Based on some physical arguments and numerical evidences, we tend to believe that a global existence of the solution can not be expected in general. We are, however, able to prove a global existence result for a modified system in one spatial dimension.

The paper is organized as follows. Section 2 contains the discussion and simplifi- 
cation of the (mathematical) governing (partial differential) equations of the system, including solution spaces. The local existence for the Lithium-ion model is proved in Section 3. Section 4 is then devoted to establish the global existence for the slightly modified model when the spatial dimension $n=1$.

2. The model equations. In this section, we will describe some details related to the system (1.1)-(1.3).

First of all, let us introduce some standard notation and terminologies.

$L^{p}(\Omega)(p>1)$ denotes the space of measurable functions $v$ defined on $\Omega$ such that $|v|^{p}$ is integrable. $L^{\infty}(\Omega)$ denotes the space of measurable functions on $\Omega$ that are bounded almost everywhere.

$$
H^{1}(\Omega)=\left\{u \in L^{2}(\Omega) \mid D u \in L^{2}(\Omega)\right\}
$$

$C^{\beta}(\bar{\Omega})(0<\beta<1)$ denotes the set of the functions $v$ such that

$$
\begin{gathered}
{[v]_{\beta, \Omega}=\sup _{x, y \in \Omega} \frac{|v(x)-v(y)|}{|x-y|^{\beta}}<\infty .} \\
C^{\beta, \beta / 2}(\bar{\Omega} \times[0, T])=\left\{u \mid[u]_{\beta, \bar{\Omega} \times[0, T]}=\sup _{(x, t),(y, \tau) \in \bar{\Omega} \times[0, T]} \frac{|u(x, t)-u(y, \tau)|}{\left(|x-y|^{2}+|t-\tau|\right)^{\beta / 2}}<\infty\right\}
\end{gathered}
$$

In (1.1), $\kappa$ represents the effective diffusional conductivity which is a (continuous) piecewise smooth function of $c$ satisfying

$$
\kappa(0)=0 ; \quad \kappa(c)>0 \text { for } c>0 .
$$

This degenerating property of $\kappa$ is one of the many complications of our model equation.

In (1.2), $\sigma$ is another effective diffusional conductivity. It is piecewise constant, positive on $\Omega^{\prime}$ but identically zero in the subdomain domain $\Omega_{s}$ (the separator). This is another degenerating feature of the system. In (1.3), both $D$ and $\varepsilon_{e}$ are positive and piecewise constant.

The function $U$ is a known bounded smooth function of $c$. The function $S_{e}$ is a nonlinear function of $\Phi_{e}, \Phi_{s}$ and $c$, taking the following form:

$$
S_{e}=\mathrm{j}= \begin{cases}\alpha_{4} c^{\frac{1}{2}} \sinh \left(\alpha_{2}\left(\Phi_{s}-\Phi_{e}-U(c)\right)\right), & \text { in } \Omega^{\prime} \\ 0, & \text { in } \Omega_{s},\end{cases}
$$

and

$$
S_{c}=\alpha_{3} \mathrm{j}=\alpha_{3} S_{e} .
$$

We note again that $S_{e}$ vanishes in the domain $\Omega_{s}$.

Finally, $\alpha_{i}(1 \leq i \leq 4)$ are all positive constants, see $[8,16]$ for the values of all the constants.

Let us now discuss the initial and boundary conditions. We first impose conditions on the 'external' boundary $\Gamma_{a} \cup \Gamma_{c}$ of $\Omega^{\prime}$ as follows:

$$
-\sigma \frac{\partial \Phi_{s}}{\partial n}=I
$$


where $I$ is a given current and $\Gamma_{a}=\partial \Omega_{a} \cap \partial \Omega, \Gamma_{c}=\partial \Omega_{c} \cap \partial \Omega$. We assume that

$$
\int_{\Gamma_{a} \cup \Gamma_{c}} I d A=0 .
$$

This means, for lithium-ion battery, no charge is generated or consumed within the battery. Since $\Phi_{s}$ is only defined in $\Omega^{\prime}$ one prescribes for $\Phi_{s}$ on the 'inner' boundaries of $\Omega^{\prime}$

$$
-\sigma \frac{\partial \Phi_{s}}{\partial n}=0, \quad \text { on }\left(\partial \Omega_{a} \cap \partial \Omega_{s}\right) \cup\left(\partial \Omega_{c} \cap \partial \Omega_{s}\right) .
$$

(no current through the separator for the solid phase).

Let $\Phi_{s}^{0} \in H^{1}\left(\Omega^{\prime}\right) \cap L^{\infty}\left(\Omega^{\prime}\right)$ that satisfies the same boundary conditions for $\Phi_{s}$, namely

$$
\frac{\partial \Phi_{s}^{0}}{\partial n}=\frac{\partial \Phi_{s}}{\partial n}, \quad x \in \partial \Omega^{\prime}
$$

and satisfies the following equation

$$
-\nabla \cdot\left(\sigma \nabla \Phi_{s}^{0}\right)=f, \quad x \in \Omega^{\prime},
$$

where $f$ be a constant function on $\Omega_{a}$ and $\Omega_{c}$ respectively, satisfying

$$
\int_{\Omega_{a}} f=\int_{\partial \Omega_{a}} I \text { and } \int_{\Omega_{c}} f=\int_{\partial \Omega_{c}} I
$$

By the choice of $f$, it is easy to see that $\Phi_{s}^{0}$ is well-defined.

For convenience, with the help of the above function $\Phi_{s}^{0}$, we make the following change of variables

$$
\Phi_{s} \leftarrow \Phi_{s}-\Phi_{s}^{0}, \quad \Phi_{e} \leftarrow \Phi_{e}-\alpha_{1} \ln c, \quad U(c) \leftarrow U(c)-\Phi_{s}^{0} .
$$

We have the following equivalent system of partial differential equations:

$$
\begin{aligned}
-\nabla \cdot\left(\kappa(c) \nabla \Phi_{e}\right)-S_{e}=0, & x \in \Omega, \\
-\nabla \cdot\left(\sigma \nabla \Phi_{s}\right)+S_{e}+f=0, & x \in \Omega^{\prime}, \\
\frac{\partial\left(\varepsilon_{e} c\right)}{\partial t}-\nabla \cdot(D \nabla c)-S_{c}=0, & (x, t) \in \Omega \times(0, T)
\end{aligned}
$$

with homogeneous Neumann boundary conditions:

$$
\begin{gathered}
\frac{\partial \Phi_{e}}{\partial n}=\frac{\partial c}{\partial n}=0, \text { for } x \in \partial \Omega, \\
\sigma \frac{\partial \Phi_{s}}{\partial n}=0, \text { for } x \in \partial \Omega^{\prime}
\end{gathered}
$$

and also the initial condition:

$$
c(x, 0)=c_{0}(x), \quad x \in \Omega,
$$

where we assume that $c_{0} \geq \epsilon_{0}>0$, and $c_{0} \in C^{0, \beta_{0}}(\bar{\Omega})$ for some $\beta_{0}>0$. 
Given $T>0$, by a weak solution of $(2.2-2.7)$, we mean a triple $\left(\Phi_{e}, \Phi_{s}, c\right)$ with

$$
\begin{gathered}
\Phi_{e} \in C\left([0, T], H^{1}(\Omega) \cap L^{\infty}(\Omega)\right), \\
\Phi_{s} \in C\left([0, T], H^{1}\left(\Omega^{\prime}\right) \cap L^{\infty}\left(\Omega^{\prime}\right)\right),
\end{gathered}
$$

and

$$
c \in C\left([0, T], H^{1}(\Omega) \cap L^{\infty}(\Omega)\right), c_{t} \in L^{\infty}\left([0, T], L^{2}(\Omega)\right), c>0
$$

satisfying

$$
\begin{gathered}
\int_{\Omega} \kappa(c) \nabla \Phi_{e} \cdot \nabla \tilde{\Phi}_{e}-\int_{\Omega} S_{e} \tilde{\Phi}_{s}=0, \quad \forall \tilde{\Phi}_{e} \in H^{1}(\Omega), t \in(0, T) \text { a.e. } \\
\int_{\Omega^{\prime}} \sigma \nabla \Phi_{s} \cdot \nabla \tilde{\Phi}_{s}+\int_{\Omega}\left(S_{e}+f\right) \tilde{\Phi}_{s}=0, \quad \forall \tilde{\Phi}_{s} \in H^{1}\left(\Omega^{\prime}\right), t \in(0, T) \text { a.e. } \\
\int_{\Omega}\left(\varepsilon_{e} c\right)_{t} \tilde{c}+\int_{\Omega} D \nabla c \cdot \nabla \tilde{c}-\int_{\Omega} S_{c} \tilde{c}=0, \\
\forall \tilde{c} \in H^{1}(\Omega) .
\end{gathered}
$$

We notice that, for any $m(t) \in C([0, T]),\left(\Phi_{e}+m(\mathrm{t}), \Phi_{s}+m(\mathrm{t})\right)$ is obviously a solution of the system $(2.2)$ and $(2.3)$, if $\left(\Phi_{e}, \Phi_{s}\right)$ is a solution.

For uniqueness, we shall assume that $\Phi_{e}$ satisfies the following condition

$$
\int_{\Omega} \Phi_{e} d x=0, \quad \forall t \in(0, T),
$$

which is necessary for uniqueness. For convenience, we define

$$
H_{*}^{1}(\Omega)=\left\{u \in H^{1}(\Omega), \int_{\Omega} u d x=0\right\},
$$

and we can define $L_{*}^{\infty}(\Omega)$ and $C_{*}^{\alpha}(\Omega)$ similarly.

3. The elliptic system for potential variables. For $M>0, T>0$ and $\beta \in\left(0, \beta_{0}\right]$, denote

$$
Z_{M}=\left\{c \in C^{\beta, \beta / 2}(\bar{\Omega} \times[0, T]) ; c \geq 1 / M,\|c\|_{C^{\beta, \beta / 2}(\bar{\Omega} \times[0, T])} \leq M\right\} .
$$

Fixing $c \in Z_{M}$, we first study the elliptic sub-system given by (2.2) and (2.3) with homogeneous Neumann boundary conditions.

3.1. Uniqueness. First we show that the solution, subject to $(2.8)$, to the elliptic system of (2.2) and (2.3) is unique. Let $\left(\Phi_{e}^{i}, \Phi_{s}^{i}\right), i=1,2$, be two solutions to the system. Set

$$
(\phi, \psi)=\left(\Phi_{e}^{1}-\Phi_{e}^{2}, \Phi_{\mathrm{s}}^{1}-\Phi_{\mathrm{s}}^{2}\right) .
$$

Multiplying the difference of the equations for $\Phi_{e}^{i}$ by $\phi$ and integrating, we obtain

$$
(\kappa(c) \nabla \phi, \nabla \phi)+\left(\phi, S_{e}^{1}-S_{e}^{2}\right)=0,
$$

where

$$
S_{e}^{1}-S_{e}^{2}=S_{e}\left(\Phi_{s}^{1}-\Phi_{e}^{1}\right)-S_{e}\left(\Phi_{s}^{2}-\Phi_{e}^{2}\right)=S_{e}^{\prime}(\xi)(\phi-\eta)
$$


for some $\xi$ between $\left(\Phi_{s}^{1}-\Phi_{e}^{1}\right)$ and $\left(\Phi_{s}^{2}-\Phi_{e}^{2}\right)$. Clearly, $S_{e}^{\prime}(\xi)$ is positive in $\Omega^{\prime}$ (zero in $\Omega_{s}$ ) by (2.1). Similarly,

$$
(\sigma \nabla \psi, \nabla \psi)-\left(\psi, S_{e}^{1}-S_{e}^{2}\right)=0 .
$$

Taking the sum we then obtain

$$
\int_{\Omega} \kappa(c)|\nabla \phi|^{2} d x+\int_{\Omega^{\prime}} \sigma|\nabla \psi|^{2} d x+\int_{\Omega^{\prime}}(\phi-\psi)^{2} S_{e}^{\prime}(\xi) d x=0
$$

It follows that

$$
\int_{\Omega} \kappa(c)|\nabla \phi|^{2}=0, \quad t \in(0, T) \text { a.e. }
$$

which, by (2.8), in turn immediately implies

$$
\phi=0, \quad \text { a.e. in } \Omega \times(0, T) .
$$

Similarly, we have

$$
\psi=0, \quad \text { a.e. in } \Omega^{\prime} \times(0, T),
$$

since now

$$
\int_{\Omega^{\prime}} \psi^{2} d x=\int_{\Omega^{\prime}}(\phi-\psi)^{2} S_{e}^{\prime}(\xi) d x=0 .
$$

This gives the uniqueness of the solution to the elliptic system.

3.2. A priori supremum estimates. The "source" term is

$$
\begin{aligned}
S_{e} & =\alpha_{4} c^{\frac{1}{2}} \sinh \left(\alpha_{2}\left(\Phi_{s}-\Phi_{e}-\alpha_{1} \ln c-U(c)\right)\right) \\
& =\alpha_{4}\left(h c^{1 / 2-d} e^{\alpha_{2}\left(\Phi_{s}-\Phi_{e}\right)}-h^{-1} c^{1 / 2+d} e^{-\alpha_{2}\left(\Phi_{s}-\Phi_{e}\right)}\right)
\end{aligned}
$$

where $d=\alpha_{1} \alpha_{2}$,

$$
\frac{1}{K} \leq h \equiv e^{-U(c)} \leq K<\infty
$$

for some fixed constant $K>0$ since that $U$ is a bounded smooth function of $c$.

For simplicity, in the following exposition, we take

$$
h=\alpha_{4}=1 .
$$

We note that the subsequent analysis without the above assumption is a little bit more complicated but can be carried out similarly.

We define

$$
X=L_{*}^{\infty}(\Omega) \times L^{\infty}\left(\Omega^{\prime}\right), \quad Y=H_{*}^{1}(\Omega) \times H^{1}\left(\Omega^{\prime}\right) .
$$

Given any $V=(u, v) \in X$ and $\delta \in[0,1]$, consider the following decoupled system,

$$
\begin{array}{cl}
\left.\int_{\Omega} \kappa(c) \nabla \Phi_{e} \cdot \nabla \phi-\int_{\Omega}\left(\delta\left(S_{e}(v-u, c)-u\right)\right)-\Phi_{e}\right) \phi=0, & \forall \phi \in H_{*}^{1}(\Omega) \\
\int_{\Omega^{\prime}} \sigma \nabla \Phi_{s} \cdot \nabla \psi+\int_{\Omega^{\prime}}\left(\delta\left(S_{e}(v-u, c)+f-v\right)+\Phi_{s}\right) \psi=0, & \forall \psi \in H^{1}\left(\Omega^{\prime}\right) .
\end{array}
$$


The existence of a solution $\Phi=\left(\Phi_{e}, \Phi_{s}\right) \in X \cap Y$ to the system of (3.3) and (3.4) is standard via a minimization argument. The uniqueness of such a solution is obvious (same as shown in 3.1.1.).

Now define a mapping

$$
Z: X \times[0,1] \rightarrow X, \quad Z((u, v), \delta)=\Phi=\left(\Phi_{e}, \Phi_{s}\right),
$$

where $\Phi=\left(\Phi_{e}, \Phi_{s}\right) \in X \cap Y$ is the unique solution to the system of (3.3) and (3.4).

Lemma 3.1. If $\Phi=Z(\Phi, \delta)$ is a solution to the elliptic system (3.3) and (3.4) with $(u, v)=\Phi=\left(\Phi_{e}, \Phi_{s}\right)$, then

$$
\begin{gathered}
\int_{\Omega} \kappa(c)\left|\nabla \Phi_{e}\right|^{2}+\int_{\Omega^{\prime}} \sigma\left|\nabla \Phi_{s}\right|^{2}+(1-\delta) \int_{\Omega^{\prime}} \Phi_{s}^{2}+(1-\delta) \int_{\Omega} \Phi_{e}^{2} \\
+\delta \int_{\Omega^{\prime}}\left|\Phi_{e}-\Phi_{s}\right|\left(c^{1 / 2-d} e^{\alpha_{2}\left(\Phi_{e}-\Phi_{s}\right)}+c^{1 / 2+d} e^{-\alpha_{2}\left(\Phi_{e}-\Phi_{s}\right)}\right)+\delta \int_{\Omega^{\prime}} f \Phi_{s} \\
\leq\left(2 / \alpha_{2}+2 e^{\alpha_{2}}\right) \int_{\Omega} H(c),
\end{gathered}
$$

where $H(c)=c^{1 / 2-d}+c^{1 / 2+d}$.

Proof. Adding (3.3) to (3.4) with $\phi=\Phi_{e}$ and $\psi=\Phi_{s}$, we obtain

$$
\begin{gathered}
\int_{\Omega} \kappa(c)\left|\nabla \Phi_{e}\right|^{2}+\int_{\Omega^{\prime}} \sigma\left|\nabla \Phi_{s}\right|^{2}+(1-\delta) \int_{\Omega^{\prime}} \Phi_{s}^{2}+(1-\delta) \int_{\Omega} \Phi_{e}^{2} \\
+\delta \int_{\Omega^{\prime}}\left(\Phi_{e}-\Phi_{s}\right) S_{e}+\delta \int_{\Omega^{\prime}} f \Phi_{s}=0 .
\end{gathered}
$$

Let

$$
G_{1}=\left\{x \in \Omega^{\prime}: \Phi_{s}-\Phi_{e} \geq 1\right\}, \quad G_{2}=\left\{x \in \Omega^{\prime}: \Phi_{s}-\Phi_{e} \leq-1\right\},
$$

and

$$
G_{3}=\left\{x \in \Omega^{\prime}:\left|\Phi_{s}-\Phi_{e}\right|<1\right\} .
$$

Thus $\Omega^{\prime}=G_{1} \cup G_{2} \cup G_{3}$. Clearly

$$
\begin{aligned}
\left|\int_{G_{3}}\left(\Phi_{s}-\Phi_{e}\right) S_{e}\right| & \leq \int_{G_{3}}\left|\left(\Phi_{s}-\Phi_{e}\right) S_{e}\right| \\
& \leq \int_{G_{3}} \alpha\left|\Phi_{s}-\Phi_{e}\right|\left(c^{1 / 2-d} e^{-\alpha_{2}\left(\Phi_{s}-\Phi_{e}\right)}+c^{1 / 2+d} e^{-\alpha_{2}\left(\Phi_{s}-\Phi_{e}\right)}\right) \\
& \leq 2 e^{\alpha_{2}} \int_{G_{3}}\left(c^{1 / 2-d}+c^{1 / 2+d}\right) \leq 2 e^{\alpha_{2}} \int_{\Omega} H(c) .
\end{aligned}
$$

On $G_{1}$, we have

$$
\left.\mid\left(\Phi_{s}-\Phi_{e}\right) e^{-\alpha_{2}\left(\Phi_{s}-\Phi_{e}\right)}\right) \mid \leq \max _{\tau \geq 1}\left(\tau e^{-\alpha_{2} \tau}\right) \leq\left(2 \alpha_{2}\right)^{-1},
$$


and in turn

$$
\begin{aligned}
\int_{G_{1}}\left(\Phi_{s}-\Phi_{e}\right) S= & \int_{G_{1}}\left(\Phi_{s}-\Phi_{e}\right)\left(e^{\alpha_{2}\left(\Phi_{s}-\Phi_{e}\right)} c^{1 / 2-d}-e^{-\alpha_{2}\left(\Phi_{s}-\Phi_{e}\right)} c^{1 / 2+d}\right) \\
= & \int_{G_{1}}\left|\Phi_{s}-\Phi_{e}\right|\left(e^{\alpha_{2}\left(\Phi_{s}-\Phi_{e}\right)} c^{1 / 2-d}+e^{-\alpha_{2}\left(\Phi_{s}-\Phi_{e}\right)} c^{1 / 2+d}\right) \\
& -2 \int_{G_{1}}\left(\Phi_{s}-\Phi_{e}\right) e^{-\alpha_{2}\left(\Phi_{s}-\Phi_{e}\right)} c^{1 / 2+d} \\
\geq & \int_{G_{1}}\left|\Phi_{s}-\Phi_{e}\right|\left(e^{\alpha_{2}\left(\Phi_{s}-\Phi_{e}\right)} c^{1 / 2-d}+e^{-\alpha_{2}\left(\Phi_{s}-\Phi_{e}\right)} c^{1 / 2+d}\right) \\
& -\frac{1}{\alpha_{2}} \int_{\Omega} c^{1 / 2+d} .
\end{aligned}
$$

Similarly, we have

$$
\begin{aligned}
\int_{G_{2}}\left(\Phi_{s}-\Phi_{e}\right) S= & \int_{G_{2}}\left(\Phi_{s}-\Phi_{e}\right)\left(e^{\alpha_{2}\left(\Phi_{s}-\Phi_{e}\right)} c^{1 / 2-d}-e^{-\alpha_{2}\left(\Phi_{s}-\Phi_{e}\right)} c^{1 / 2+d}\right) \\
\geq & \int_{G_{2}}\left|\Phi_{s}-\Phi_{e}\right|\left(e^{\alpha_{2}\left(\Phi_{s}-\Phi_{e}\right)} c^{1 / 2-d}+e^{-\alpha_{2}\left(\Phi_{s}-\Phi_{e}\right)} c^{1 / 2+d}\right) \\
& -\frac{1}{\alpha_{2}} \int_{\Omega} c^{1 / 2-d} .
\end{aligned}
$$

Combining the above three inequalities, we have,

$$
\begin{aligned}
\int_{\Omega^{\prime}}\left(\Phi_{s}-\Phi_{e}\right) S= & \int_{G_{1}}\left(\Phi_{s}-\Phi_{e}\right) S+\int_{G_{2}}\left(\Phi_{s}-\Phi_{e}\right) S+\int_{G_{3}}\left(\Phi_{s}-\Phi_{e}\right) S \\
\geq & \int_{\Omega^{\prime}}\left|\Phi_{s}-\Phi_{e}\right|\left(e^{\alpha_{2}\left(\Phi_{s}-\Phi_{e}\right)} c^{1 / 2-d}+e^{-\alpha_{2}\left(\Phi_{s}-\Phi_{e}\right)} c^{1 / 2+d}\right) \\
& -\left(\frac{2}{\alpha_{2}}+2 e^{\alpha_{2}}\right) \int_{\Omega} H(c) .
\end{aligned}
$$

Combining (3.5) and the above inequalities, we complete the proof. $\square$

Next we shall use the classical Moser iteration $[14,15]$ to establish a priori supremum estimates for $\Phi=\left(\Phi_{e}, \Phi_{s}\right) \in X \cap Y$. First, we prove the following uniform $L^{2}$-estimates.

Lemma 3.2. For $M>0$, let $c \in Z_{M}$ and $\Phi=\left(\Phi_{e}, \Phi_{s}\right) \in X \cap Y$ be a solution of the equation $\Phi=Z(\Phi, \delta)$ for some $\delta \in[0,1]$. Then there exists $\alpha(M)>0$ such that

$$
\int_{\Omega} \Phi_{e}^{2}+\int_{\Omega^{\prime}} \Phi_{s}^{2} \leq \alpha(M)
$$

and

$$
\delta\left|\int_{\Omega^{\prime}} S_{e}\right| \leq \alpha(M)
$$

Proof. Obviously $\Phi=Z(\Phi, \delta)$ is a solution to the elliptic system (3.3) and (3.4) 
with $(u, v)=\Phi=\left(\Phi_{e}, \Phi_{s}\right)$. Then

$$
\begin{gathered}
\int_{\Omega} \kappa(c)\left|\nabla \Phi_{e}\right|^{2}+\int_{\Omega^{\prime}} \sigma\left|\nabla \Phi_{s}\right|^{2}+(1-\delta) \int_{\Omega^{\prime}} \Phi_{s}^{2}+(1-\delta) \int_{\Omega} \Phi_{e}^{2} \\
+\delta \int_{\Omega^{\prime}}\left|\Phi_{e}-\Phi_{s}\right|\left(c^{1 / 2-d} e^{\alpha_{2}\left(\Phi_{e}-\Phi_{s}\right)}+c^{1 / 2+d} e^{-\alpha_{2}\left(\Phi_{e}-\Phi_{s}\right)}\right)+\delta \int_{\Omega^{\prime}} f \Phi_{s} \\
\leq\left(2 / \alpha_{2}+2 e^{\alpha_{2}}\right) \int_{\Omega} H(c) .
\end{gathered}
$$

In the rest of the proof, we should use the special notation $\lesssim$ and $\gtrsim \cdot x \lesssim y$ means $x \leq \beta(M) y$ for some positive constant $\beta(M)$ depending on $M$ similarly $x \gtrsim y$ means $x \geq \gamma(M) y$ for some positive constant $\gamma(M)$ depending on $M$.

Evidently

$$
\left|\Phi_{e}-\Phi_{s}\right|\left(e^{\alpha_{2}\left(\Phi_{e}-\Phi_{s}\right)}+e^{-\alpha_{2}\left(\Phi_{e}-\Phi_{s}\right)}\right) \gtrsim\left|\Phi_{e}-\Phi_{s}\right|^{2} .
$$

Using the fact that $c \in Z_{M}$, we have

$$
1 \lesssim \sigma, c^{1 / 2-d}, c^{1 / 2+d}, H(c), \kappa(c) \lesssim 1
$$

It follows that

$$
\int_{\Omega} \kappa(c)\left|\nabla \Phi_{e}\right|^{2}+\sigma \int_{\Omega^{\prime}}\left|\nabla \Phi_{s}\right|^{2} \gtrsim \int_{\Omega}\left|\nabla \Phi_{e}\right|^{2}+\int_{\Omega^{\prime}}\left|\nabla \Phi_{s}\right|^{2},
$$

and

$$
\int_{\Omega^{\prime}}\left|\Phi_{e}-\Phi_{s}\right|\left(c^{1 / 2-d} e^{\alpha_{2}\left(\Phi_{e}-\Phi_{s}\right)}+c^{1 / 2+d} e^{-\alpha_{2}\left(\Phi_{e}-\Phi_{s}\right)}\right) \gtrsim \int_{\Omega^{\prime}}\left|\Phi_{e}-\Phi_{s}\right|^{2} .
$$

It follows that

$$
\begin{gathered}
\int_{\Omega}\left|\nabla \Phi_{e}\right|^{2}+\int_{\Omega^{\prime}}\left|\nabla \Phi_{s}\right|^{2}+\delta \int_{\Omega^{\prime}}\left|\Phi_{e}-\Phi_{s}\right|^{2} \\
+(1-\delta) \int_{\Omega^{\prime}} \Phi_{s}^{2}+(1-\delta) \int_{\Omega} \Phi_{e}^{2} \lesssim\left(1+\left|\int_{\Omega^{\prime}} f \Phi_{s}\right|\right) .
\end{gathered}
$$

By (2.8) and the Poincaré's inequality,

$$
\int_{\Omega} \Phi_{e}^{2}=\lesssim \int_{\Omega}\left|\nabla \Phi_{e}\right|^{2} \lesssim 1
$$

We now write $\phi_{s}=\delta\left(\phi_{s}-\phi_{e}\right)+(1-\delta) \phi_{s}+\delta \phi_{e}$. It follows from (3.5) again

$$
\begin{gathered}
\quad \frac{1}{3} \int_{\Omega^{\prime}} \Phi_{s}^{2} \leq \delta \int_{\Omega^{\prime}}\left|\Phi_{e}-\Phi_{s}\right|^{2}+(1-\delta) \int_{\Omega^{\prime}} \Phi_{s}^{2}+\delta \int_{\Omega} \Phi_{e}^{2} \\
\lesssim \quad\left(1+\left|\int_{\Omega^{\prime}} f \Phi_{s}\right|\right) \lesssim \int_{\Omega^{\prime}} \Phi_{s}^{2}+\int_{\Omega^{\prime}} f^{2} \lesssim 1 .
\end{gathered}
$$

Finally, taking $\psi=1$ in (3.4) yields

$$
\delta\left|\int_{\Omega^{\prime}} S_{e}\right| \leq(1-\delta)\left|\int_{\Omega^{\prime}} \Phi_{s}\right|+\delta\left|\int_{\Omega^{\prime}} f \Phi_{s}\right| \lesssim 1
$$


The proof is complete.

With the aid of Lemma 3.2, we are in a position to derive the following a priori estimates, independent of both $\delta$ and the solution $\Phi=\left(\Phi_{e}, \Phi_{s}\right) \in X \cap Y$. In the following, $\alpha(M)$ denotes a generous constant which depends on $M$.

Lemma 3.3. Let $\Phi=\left(\Phi_{e}, \Phi_{s}\right) \in X \cap Y$ be a solution of $\Phi=Z(\Phi, \delta)$ for some $\delta \in[0,1]$. Then there exists a positive constant $\alpha(M)$ such that

$$
\left\|\Phi_{e}\right\|_{L^{\infty}(\Omega)} \leq \alpha(M) ; \quad\left\|\Phi_{s}\right\|_{L^{\infty}\left(\Omega^{\prime}\right)} \leq \alpha(M) .
$$

Proof. For $x_{0} \in \Omega^{\prime}$, let $R>0$ such that $R<\operatorname{dist}\left(x_{0}, \partial \Omega^{\prime}\right)$. Put $B_{R}=B_{R}\left(x_{0}\right)$, the ball centered at $x_{0}$ with radius $R$. Let $\xi \in C_{0}^{\infty}\left(B_{R}\left(x_{0}\right)\right)$ be a standard cut-off function with $\xi \equiv 1$ on $B_{R / 2}$. Given any even integer $p>1$, we use the test function

$$
\phi=\xi^{2} \Phi_{e}^{p-1}-\frac{1}{|\Omega|} \int_{\Omega} \xi^{2} \Phi_{e}^{p-1} \in H_{*}^{1}
$$

in $(3.3)\left((u, v)=\left(\Phi_{e}, \Phi_{s}\right)\right)$ to obtain

$$
\begin{gathered}
\left.\int_{B_{R}} \kappa(c) \nabla \Phi_{e} \cdot \nabla\left(\xi^{2} \Phi_{e}^{p-1}\right)-\int_{B_{R}}\left(\delta S_{e}-(1-\delta) \Phi_{e}\right)\right) \xi^{2} \Phi_{e}^{p-1} \\
+\frac{\delta}{|\Omega|} \int_{\Omega} \xi^{2} \Phi_{e}^{p-1} \int_{\Omega^{\prime}} S_{e}=0
\end{gathered}
$$

Similarly, using $\psi=\xi^{2} \Phi_{s}^{p-1}$ in (3.4)

$$
\int_{B_{R}} \sigma \nabla \Phi_{s} \cdot \nabla\left(\xi^{2} \Phi_{s}^{p-1}\right)+\int_{B_{R}}\left(\delta S_{e}+\delta f+(1-\delta) \Phi_{s}\right) \xi^{2} \Phi_{s}^{p-1}=0 .
$$

Taking the sum, we then obtain

$$
\begin{aligned}
& \int_{B_{R}} \kappa(c) \nabla \Phi_{e} \cdot \nabla\left(\xi^{2} \Phi_{e}^{p-1}\right)+\int_{B_{R}} \sigma \nabla \Phi_{s} \cdot \nabla\left(\xi^{2} \Phi_{s}^{p-1}\right) \\
& +\delta \int_{B_{R}} S_{e} \xi^{2}\left(\Phi_{s}^{p-1}-\Phi_{e}^{p-1}\right)+(1-\delta) \int_{B_{R}} \xi^{2}\left(\Phi_{s}^{p}+\Phi_{e}^{p}\right) \\
& +\frac{\delta}{|\Omega|} \int_{\Omega} \xi^{2} \Phi_{e}^{p-1} \int_{\Omega^{\prime}} S_{e}+\delta \int_{B_{R}} f \xi^{2} \Phi_{s}^{p-1}=0 .
\end{aligned}
$$

Using (2.1) once more, we have

$$
S_{e}\left(\Phi_{s}-\Phi_{e}, c\right)=S_{e}(0, c)+S_{e}^{\prime}(\eta, c)\left(\Phi_{s}-\Phi_{e}\right),
$$

where $S_{e}^{\prime}(\eta, c) \geq 0$. It follows that

$$
\begin{aligned}
& \int_{B_{R}} S_{e}\left(\Phi_{s}-\Phi_{e}, c\right) \xi^{2}\left(\Phi_{s}^{p-1}-\Phi_{e}^{p-1}\right) d x \\
\geq & -\alpha(M) \int_{B_{R}} \xi^{2}\left(\left|\Phi_{e}\right|^{p-1}+\left|\Phi_{s}\right|^{p-1}\right) d x
\end{aligned}
$$

since (recall $p$ even)

$$
\left(\Phi_{s}^{p-1}-\Phi_{e}^{p-1}\right)\left(\Phi_{s}-\Phi_{e}\right) \geq 0
$$




$$
\int_{B_{R}} f \xi^{2} \Phi_{s}^{p-1} \leq\|f\|_{L^{\infty}} \int_{B_{R}} \xi^{2}\left|\Phi_{s}\right|^{p-1}
$$

By Lemma 3.2, we have

$$
\delta \int_{\Omega} \xi^{2} \Phi_{e}^{p-1} \int_{\Omega^{\prime}} S_{e} \geq-\alpha(M) \int_{B_{R}} \xi^{2}\left|\Phi_{e}\right|^{p-1} .
$$

Therefore

$$
\begin{aligned}
& \int_{B_{R}} \xi^{2}\left|\nabla \Phi_{e}^{p / 2}\right|^{2}+\int_{B_{R}} \xi^{2}\left|\nabla \Phi_{s}^{p / 2}\right|^{2} \\
\leq & \alpha(M)\left(\int_{B_{R}}|\nabla \xi|^{2}\left(\Phi_{e}^{p}+\Phi_{s}^{p}\right)+p \int_{B_{R}} \xi^{2}\left(\left|\Phi_{e}\right|^{p-1}+\left|\Phi_{s}\right|^{p-1}\right)+p^{2} \int_{B_{R}} \xi^{2}\left|\Phi_{s}\right|^{p-2}\right) .
\end{aligned}
$$

With the above estimate, we can use a standard argument from the classical NashMoser-De Giorgi boot-strap to show that there exists $\alpha(M)>0$ such that (see [7, 11] for details)

$$
\sup _{B_{R / 2}}\left|\Phi_{e}\right| \leq \frac{\alpha(M)}{\left|B_{R}\right|} \int_{B_{R}}\left|\Phi_{e}\right|+\frac{\alpha(M)}{\left|B_{R}\right|} \int_{B_{R}}\left|\Phi_{s}\right|
$$

and

$$
\sup _{B_{R / 2}}\left|\Phi_{s}\right| \leq \frac{\alpha(M)}{\left|B_{R}\right|} \int_{B_{R}}\left|\Phi_{e}\right|+\frac{\alpha(M)}{\left|B_{R}\right|} \int_{B_{R}}\left|\Phi_{s}\right| .
$$

Clearly the above estimates are valid on all of $\Omega$ (including the boundary) for $\Phi_{e}$ and $\Omega^{\prime}$ for $\Phi_{s}$, in view of the homogeneous Neumann boundary conditions. Namely, $\forall x_{0} \in \Omega$ and $R \leq 1$, we have

$$
\sup _{B_{R / 2}\left(x_{0}\right) \cap \Omega}\left|\Phi_{e}\right| \leq \alpha(M)\left(\int_{\Omega}\left|\Phi_{e}\right|+\int_{\Omega^{\prime}}\left|\Phi_{s}\right|\right)
$$

and

$$
\sup _{B_{R / 2}\left(x_{0}\right) \cap \Omega^{\prime}}\left|\Phi_{s}\right| \leq \alpha(M)\left(\int_{\Omega}\left|\Phi_{e}\right|+\int_{\Omega^{\prime}}\left|\Phi_{s}\right|\right) .
$$

Now the proof follows directly from Lemma 3.2.

3.3. Existence using Leray-Schauder theory. Now, for $t \in(0, T)$, we shall prove the existence of a solution (in $H_{*}^{1} \times H^{1}$ ) to the system of (2.2) and (2.3) using the Leray-Schauder theorem (see Theorem 11.6, p.280, [7]). Specifically, we want to show that $V=Z(V, 1)(V \in X)$ has a fixed point in the Banach space $X$. To this end, let us first state and verify the following properties of $Z$ :

1. $Z(V, 0)=0$ for all $V \in X$. This is obvious.

2. $Z: X \rightarrow X$ is compact. Let $\Phi=Z(V, \delta)$. That is, $\Phi$ satisfies the system (3.3) and (3.4). A standard De-Giorgi estimate shows that $\Phi \in C_{*}^{\beta_{1}}(\Omega) \times C^{\beta_{1}}\left(\Omega^{\prime}\right)$ for some $\beta_{1}>0$ (independent of $\beta$ ). The claim follows by the fact that the imbedding $C_{*}^{\beta_{1}} \times C^{\beta_{1}} \longrightarrow X$ is compact.

3. There exists a positive constant $\alpha(M)$ (independent of $\delta$ and $W$ ) such that

$$
\|W\|_{X} \leq \alpha, \quad \forall W \in\{V \in X \mid \exists \delta \in[0,1], V=Z(V, \delta)\} .
$$

This is the estimate proved in Lemma 3.3. 
Thus by the Leray-Schauder theorem, the mapping $V=Z(V, 1)$ has a fix point $\Phi$ in $X$.

Theorem 3.4. Let $\beta \in\left(0, \beta_{0}\right], M>0$ and $T>0$. Suppose $c \in Z_{M}$. Then for any $t \in(0, T)$, the system of (2.2) and (2.3), with homogeneous Neumann boundary condition, admits a unique solution $\Phi=\left(\Phi_{e}, \Phi_{s}\right) \in X \cap Y$. Moreover, there exists $\alpha(M)>0$ such that

$$
\left\|\Phi_{e}\right\|_{L^{\infty}(\Omega)}+\left\|\Phi_{s}\right\|_{L^{\infty}\left(\Omega^{\prime}\right)} \leq \alpha(M) .
$$

Proof. The fixed point $\Phi=\left(\Phi_{e}, \Phi_{s}\right) \in X \cap Y$ of $x=Z(x, 1)$ obtained above clearly satisfies the system of (3.3) and (3.4) with $(u, v)=\Phi=\left(\Phi_{e}, \Phi_{s}\right)$ and $\delta=1$. Moreover, there exists $\alpha(M)>0$ such that

$$
\left\|\Phi_{e}\right\|_{L^{\infty}(\Omega)}+\left\|\Phi_{s}\right\|_{L^{\infty}\left(\Omega^{\prime}\right)} \leq \alpha(M) .
$$

Clearly there exists a constant $\alpha(M)$ such that

$$
\int_{\Omega^{\prime}} S_{e}\left(\Phi_{s} \pm \alpha(M)-\Phi_{e}, c\right) d x=0,
$$

in view of the definition (2.1), since

$$
c, c^{-1},\left|\Phi_{s}\right|,\left|\Phi_{e}\right| \leq \alpha(M) .
$$

$\Phi=\left(\Phi_{e}, \Phi_{s} \pm \alpha(M)\right)$ is the desired solution to the system (2.2) and (2.3) with homogeneous Neumann boundary conditions.

We close this section by giving the following regularity of $\Phi$ in $t$.

Lemma 3.5. Let $\beta \in\left(0, \beta_{0}\right], M>0$ and $T>0$. Suppose $c \in Z_{M}$ and $\kappa \in C^{\gamma}$ for some $\gamma \in(0,1)$. Then the unique solution $\Phi=\left(\Phi_{e}, \Phi_{s}\right) \in X \cap Y$ given in Theorem 3.4 satisfies

$$
\Phi \in C^{\gamma^{\prime}}\left([0, T], H_{*}^{1}(\Omega) \times H^{1}\left(\Omega^{\prime}\right)\right),
$$

for some $\gamma^{\prime} \in(0,1)$.

Remark. The exponent $\gamma^{\prime}$ depends on the Hölder exponents $\beta$ and $\gamma$.

Proof. The proof is essentially the same as the uniqueness argument given in 4.1.1. Indeed, for $t_{i} \in(0, T), i=1,2$, put

$$
\Phi_{e}^{i}=\Phi_{e}\left(x, t_{i}\right), \quad \Phi_{s}^{i}=\Phi_{s}\left(x, t_{i}\right), \quad c_{i}=c\left(x, t_{i}\right),
$$

and

$$
\phi=\Phi_{e}^{1}-\Phi_{e}^{2} \quad \psi=\Phi_{s}^{1}-\Phi_{s}^{2} .
$$

Similarly as in 3.1.1, we have

$$
\begin{gathered}
\int_{\Omega} \kappa\left(c_{1}\right)|\nabla \phi|^{2}+\int_{\Omega^{\prime}} \sigma|\nabla \psi|^{2}+\int_{\Omega^{\prime}}(\phi-\psi)^{2} S_{e}^{\prime}(\xi) \\
+\int_{\Omega^{\prime}}(\phi-\psi)^{2}\left(S_{e}\left(\Phi_{s}^{2}-\Phi_{e}^{2}, c_{2}\right)-S_{e}\left(\Phi_{s}^{2}-\Phi_{e}^{2}, c_{1}\right)\right) \\
+\int_{\Omega}\left(\kappa\left(c_{1}\right)-\kappa\left(c_{2}\right)\right) \nabla \phi \nabla \Phi_{e}^{2}=0 .
\end{gathered}
$$

Now the conclusion follows from Theorem 3.4, Lemmas 3.2-3.3 and their proofs, and the assumptions. 
4. Local existence. In this section, we shall prove that the system (1.1-1.3) poses a unique solution upto certain time $t^{*}$ under some proper initial and boundary value conditions.

Now we will prove a local (in time) existence result for the system of (2.2), (2.3) and (2.4). Set

$$
M=2\left\|c_{0}\right\|_{L^{\infty}}+2\left\|1 / c_{0}\right\|_{L^{\infty}} .
$$

For $\beta \in\left(0, \beta_{0}\right]$ and $T>0$, let $Z_{M}$ be the set given in the beginning of this section. Clearly $Z_{M}$ is a nonempty, closed, bounded and convex subset of the Banach space $C^{\beta, \beta / 2}(\bar{\Omega} \times[0, T])$.

For $v \in Z_{M}$, by Theorem 3.4 and Lemma 3.5, the system of (2.2) and (2.3) has a unique solution $\Phi=\left(\Phi_{e}, \Phi_{s}\right) \in X \cap Y$ satisfying

$$
\left\|\Phi_{e}\right\|_{L^{\infty}(\Omega)}+\left\|\Phi_{s}\right\|_{L^{\infty}\left(\Omega^{\prime}\right)} \leq \alpha(M)
$$

and for some $\gamma^{\prime} \in(0,1)$

$$
\Phi \in C^{\gamma^{\prime}}\left([0, T], H_{*}^{1}(\Omega) \times H^{1}\left(\Omega^{\prime}\right)\right) .
$$

One can solve the parabolic initial-boundary value problem (2.4) (with $S_{c}=$ $\left.S_{c}(\Phi, v)\right),(2.5)$ and $(2.7)$ in $C\left([0, T], H^{1}(\Omega)\right)$.

A classical Hölder estimate (cf. [10]) $)^{1}$ shows that there exists $\beta_{1} \in\left(0, \beta_{0}\right]$ (depending only on $\epsilon_{e}$ and $\left.D\right)$ such that $c \in C^{\beta_{1}, \beta_{1} / 2}(\bar{\Omega} \times[0, T])$ satisfying

$$
\left\|c-c_{0}\right\|_{C^{\beta, \beta / 2}} \leq \alpha(M) T^{\beta / 2}
$$

for all $\beta \leq \beta_{1}$. Now fix

$$
\beta \in\left(0, \min \left[\beta_{0}, \beta_{1}\right]\right)
$$

and define a mapping

$$
\mathbf{T}: Z_{M} \longrightarrow C^{\beta, \beta / 2}(\bar{\Omega} \times[0, T]), \quad \mathbf{T}(v)=c .
$$

Clearly $\mathbf{T}$ is continuous. Take

$$
T_{0}^{\beta / 2}=\alpha^{-1}(M) \min \{M / 2,1 / M\} .
$$

Then for $T \in\left(0, T_{0}\right]$

$$
\|c\|_{C^{\beta, \beta / 2}(\bar{\Omega} \times[0, T])} \leq\left\|c_{0}\right\|_{C^{\beta}}+\alpha(M) T_{0}^{\beta / 2} \leq M / 2+M / 2=M
$$

and

$$
c \geq c_{0}-\alpha(M) T_{0}^{\beta / 2} \geq 2 / M-1 / M=1 / M .
$$

It follows that

$$
\mathbf{T}: Z_{M} \longrightarrow Z_{M}
$$

\footnotetext{
${ }^{1}$ Initial-Boundary (homogeneous Dirichlet) value problems were treated in [10]. But similar arguments apply to Initial-Boundary (homogeneous Neumann) value problems
} 

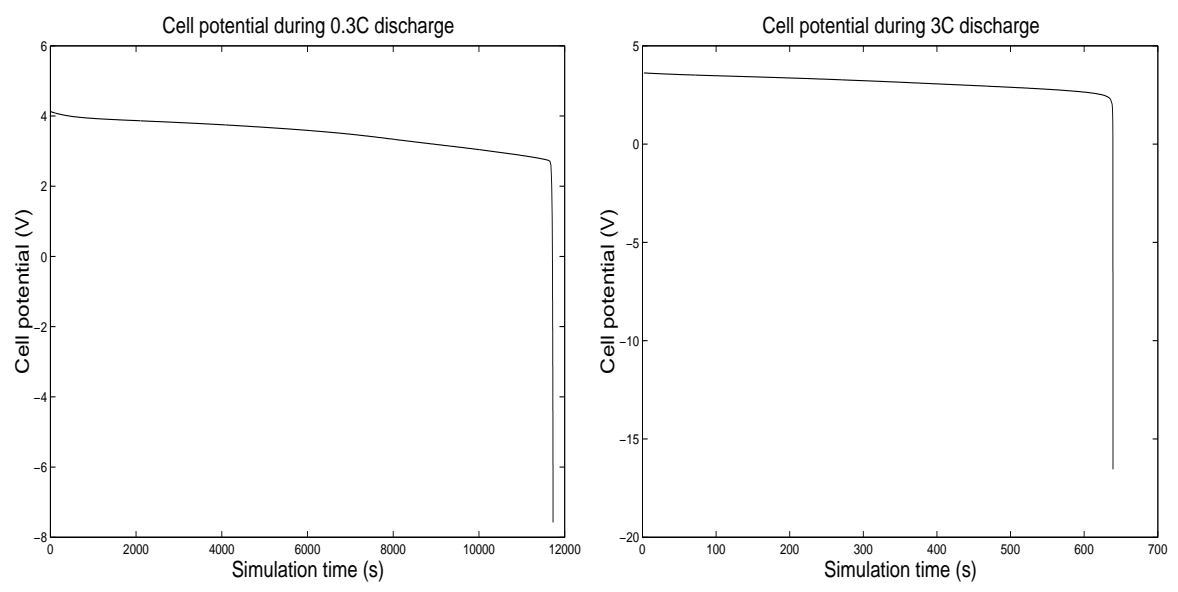

for all $T \in\left(0, T_{0}\right]$ and $\beta \in\left(0, \min \left[\beta_{0}, \beta_{1}\right]\right)$. Finally, note that $\mathbf{T}$ is also compact since $\beta<\beta_{1}$.

Therefore $\mathbf{T}$ has a fixed point $c \in Z_{M}$ by the Schauder fixed point theorem, provided $T \in\left(0, T_{0}\right]$ and $\beta \in\left(0, \min \left[\beta_{0}, \beta_{1}\right]\right)$. Clearly $\left(\Phi_{e}, \Phi_{s}, c\right)$ is a solution to $(2.2)$, $(2.3),(2.4)$.

THEOREM 4.1. There exists $T>0$ such that the system of the equations (2.2), (2.3) and (2.4) with homogeneous Neumann boundary conditions admits a solution $\left(\Phi_{e}, \Phi_{s}, c\right)$ where $\Phi_{e}$ satisfies (2.8).

5. Some remarks on the local existence. Naturally, one wishes to know whether the local solutions obtained can be extended globally (in time). From the physical point of view, a battery can last only for a limited period of time. In the model under consideration, it is assumed that the battery is either only being (continuously) discharged $(I>0)$ or charged $(I<0)$. Hence the battery is expected to be either drawn out (in the former case) or to be blown-up (in the latter case) within a finite amount of time. Furthermore, our numerical examples tend to support a finite time extinction theory. The following figures give the numerical results for $i=0.3 C=$ $0.678 \mathrm{~A}$ and $i=3 C=6.78 \mathrm{~A}$, where the current $i=\int_{\Gamma_{c}} I d A$ is the major input variable in the model.

In each of the above, we observe a sharp change of value of cell potential at a critical time and the solution cease to exist beyond this time. As expected, the battery life expectance gets shorter when the current $i$ gets larger. The following tabular shows the relationship between $i$ and the critical time $T^{*}$ during discharge.

Current and critical time

\begin{tabular}{|c|c|c|c|c|c|}
\hline Current $(i)$ & $0.3 \mathrm{C}$ & $1 \mathrm{C}$ & $1.5 \mathrm{C}$ & $2 \mathrm{C}$ & $3 \mathrm{C}$ \\
\hline$T^{*}(\mathrm{~s})$ & 11732.91 & 3332.934 & 2132.906 & 1523.385 & 639.0287 \\
\hline$i \times T^{*}$ & 3519.873 & 3332.934 & 3199.359 & 3046.770 & 1917.086 \\
\hline
\end{tabular}

However, it is not clear if a global existence (in time) for the above model can be established mathematically. Indeed, the system of partial differential equations is only a model for the reality and the above observations may not necessarily imply a finite time extinction mathematically. Nevertheless, it would be interesting to show the existence of a critical (finite extinction) time mathematically. On the other hand, 
the model assumes that the diffusion coefficient $\sigma$ identically zero in the separator region. That is, one considers the separator an absolute insulator. Yet a (small) diffusion is always present in the separator (typically $\sigma \sim 10^{-9}$ in $\Omega_{s}$ which can of course be assumed zero in practice). This, however, suggests that a diffusion (of $\Phi_{s}$ ) indeed presents in the entire battery. That is, $\sigma$ is a positive piece-wise constant in the whole domain $\Omega$ (at least mathematically). In particular, the equation of $\Phi_{s}$ is non-degenerate. Based on this observation, we shall study a slightly modified system in which $\sigma$ is positive piece-wise constant in the entire domain $\Omega$. When the spatial dimension $n=1$, we derive supremum estimates of $\Phi_{e}$ and $\Phi_{s}$ (uniform for $t \in(0, T)$ ), independent of the concentration $c$. We then establish $L^{p}$-estimates for $c$ and $c^{-1}$ for some $p$ sufficiently large. As a result, one bounds $c$ away from both infinity (upper bound on growth) and the origin (lower bound on decay) by the classical Nash-MoserDeGiorgi boot strap. The desired global existence follows by a continuation argument.

6. Global existence of a modified system. Whether the system of our partial differential equations admits a global solution in time is of great interest, particularly from a theoretical point of view.

As mentioned in the introduction, we are not able to show that the current model admits a global solution in time. A crucial reason is that it is assumed that there is no diffusion in the separator, i.e., $\sigma \equiv 0$ in $\Omega_{s}$. This assumption is practically sound and the local existence theory obtained in Section 3 is rather satisfying. Indeed, $\sigma \sim 10^{-9}$ in $\Omega_{s}$ (practically zero) and a battery can last for only a limited period of time. From a mathematical point of view, however, the fact $\sigma \sim 10^{-9}$ in $\Omega_{s}$ asserts that $\sigma>0$ is a piece-wise constant in the entire domain $\Omega$. For convenience, we make the following change of variables from $(1.1,1.2,1.3)$

$$
\Phi_{s} \leftarrow \Phi_{s}-\Phi_{s}^{0}, \quad \Phi_{e} \leftarrow \Phi_{e}-\alpha_{1} \ln c, \quad U(c) \leftarrow U(c)-\Phi_{s}^{0}
$$

where $\Phi$ is a bounded function which satisfies

$$
-\nabla \cdot\left(\sigma \nabla \Phi_{s}^{0}\right)=0, \quad x \in \Omega,
$$

with same boundary conditions for $\Phi_{s}$. Consequently, our system of partial differential equations reads

$$
\begin{gathered}
-\frac{\partial}{\partial x}\left(\kappa(c) \frac{\partial \Phi_{e}}{\partial x}\right)-S_{e}=0, \quad x \in \Omega, \\
-\frac{\partial}{\partial x}\left(\sigma \frac{\partial \Phi_{s}}{\partial x}\right)+S_{e}=0, \quad x \in \Omega \\
\frac{\partial\left(\varepsilon_{e} c\right)}{\partial t}-\frac{\partial}{\partial x}\left(D \frac{\partial c}{\partial x}\right)-S_{c}=0, \quad(x, t) \in \Omega \times(0, T)
\end{gathered}
$$

with homogeneous Neumann boundary conditions:

$$
\frac{\partial \Phi_{e}}{\partial n}=\sigma \frac{\partial \Phi_{s}}{\partial n}=\frac{\partial c}{\partial n}=0, \text { for } x \in \partial \Omega ;
$$

and also the initial condition:

$$
c(x, 0)=c_{0}(x), \quad x \in \Omega .
$$

In this section, we show that the slightly modified system (6.1-6.5) admits a global solution in time. Due to technicality, we only consider the one dimensional case $n=1$. 
For $0<L_{a s}<L_{s c}<L$, denote

$$
\Omega=(0, L), \quad \Omega_{a}=\left(0, L_{a s}\right), \quad \Omega_{s}=\left(L_{a s}, L_{s c}\right), \quad \Omega_{c}=\left(L_{s c}, L\right) .
$$

To ensure uniqueness for the system (6.1-6.5), without loss of generality, we shall impose the following condition

$$
\bar{\Phi}_{s}=\frac{1}{L} \int_{\Omega} \Phi_{s} d x=0
$$

Given $T>0$, by a weak solution of (6.1-6.5), we mean a triple $\left(\Phi_{e}, \Phi_{s}, c\right)$ with

$$
\begin{gathered}
\Phi_{e} \in C\left([0, T], H^{1}(\Omega)\right), \quad \Phi_{s} \in C\left([0, T], H^{1}(\Omega)\right), \\
c \in C\left([0, T], H^{1}(\Omega)\right), c_{t} \in L^{\infty}\left([0, T], L^{2}(\Omega)\right), c>0
\end{gathered}
$$

satisfying

$$
\begin{gathered}
\int_{\Omega} \kappa(c) \frac{\partial \Phi_{e}}{\partial x} \frac{\partial \tilde{\Phi}_{e}}{\partial x}-\int_{\Omega} S_{e} \tilde{\Phi}_{e}=0, \quad \forall \tilde{\Phi}_{e} \in H^{1}(\Omega), t \in(0, T) \text { a.e. } \\
\int_{\Omega} \sigma \frac{\partial \Phi_{s}}{\partial x} \frac{\partial \tilde{\Phi}_{s}}{\partial x}+\int_{\Omega} S_{e} \tilde{\Phi}_{s}=0, \quad \forall \tilde{\Phi}_{s} \in H^{1}(\Omega), t \in(0, T) \text { a.e. } \\
\varepsilon_{e} \int_{0}^{T} \int_{\Omega} c_{t} \tilde{c}+D \int_{0}^{T} \int_{\Omega} \frac{\partial c}{\partial x} \frac{\partial \tilde{c}}{\partial x}-\int_{0}^{T} \int_{\Omega} S_{c} \tilde{c}=0 \\
\forall \tilde{c} \in C\left([0, T], H^{1}(\Omega) \cap L^{\infty}(\Omega)\right) .
\end{gathered}
$$

6.1. Preliminary results. We first begin with technical lemmas. In the sequel, $M$ will denote generic constants depending only on $T, K, \alpha_{i}^{\prime} s$ and the domain $\Omega$. Without loss of generality, in the following exposition, we also take (3.1,3.2). Therefore

$$
S_{e}=S_{c}=S=c^{1 / 2-d} e^{\alpha_{2}\left(\Phi_{s}-\Phi_{e}\right)}-c^{1 / 2+d} e^{-\alpha_{2}\left(\Phi_{s}-\Phi_{e}\right)},
$$

where $d=\alpha_{1} \alpha_{2}$ for simplicity.

Lemma 6.1. For $t \in(0, T)$ a.e., we have

$$
\begin{aligned}
& \int_{\Omega} \sigma\left(\Phi_{s}^{\prime}\right)^{2} d x+\int_{\Omega} \kappa(c)\left(\Phi_{e}^{\prime}\right)^{2} d x \\
& \quad+\int_{\Omega}\left|\Phi_{s}-\Phi_{e}\right|\left(c^{1 / 2-d} e^{\alpha_{2}\left(\Phi_{s}-\Phi_{e}\right)}+c^{1 / 2+d} e^{-\alpha_{2}\left(\Phi_{s}-\Phi_{e}\right)}\right) \\
& \leq\left(2 e_{2}^{\alpha}+2 / \alpha_{2}\right) \int_{\Omega} H(c) d x
\end{aligned}
$$

where the derivative is with respect to $x$ and

$$
H(c)=c^{1 / 2-d}+c^{1 / 2+d} .
$$

Proof. The proof is similar as that of Lemma 3.1.

Lemma 6.2. Suppose $d=\alpha_{1} \alpha_{2}>1 / 2$ and there exists $A>0$ such that

$$
\kappa(c) c^{1 / 2-d-2} \leq A, \quad 0<c \leq 1 .
$$


Then there exists $M>0$ such that

$$
\varepsilon_{e} \frac{d}{d t} \int_{\Omega} c^{1 / 2-d} d x+\frac{\left(4 d^{2}-1\right) D}{8} \int_{\Omega} c^{-d-3 / 2}\left|c^{\prime}\right|^{2} d x \leq M \int_{\Omega} H(c) d x .
$$

Proof. For $t \in(0, T)$ and fixed $k>0$, taking $\tilde{c}=c^{-k-1}$ in (6.9) we obtain

$$
-\frac{\varepsilon_{e}}{k} \frac{d}{d t} \int_{\Omega} c^{-k} d x-(k+1) D \int_{\Omega} c^{-k-2}\left|c^{\prime}\right|^{2} d x=\int_{\Omega} c^{-k-1} S .
$$

Using the test function $\tilde{\Phi}_{e}=c^{-k-1}$ in (6.7), we derive

$$
\begin{aligned}
& \left|\int_{\Omega} c^{-k-1} S\right|=(k+1)\left|\int_{\Omega} \kappa(c) c^{-k-2} \Phi_{e}^{\prime} c^{\prime}\right| \\
\leq & M(k, D, A) \int_{\Omega} \kappa(c)\left(\Phi_{e}^{\prime}\right)^{2} d x+\frac{(k+1) D}{2 A} \int_{\Omega} \kappa(c) c^{-2 k-4}\left|c^{\prime}\right|^{2} d x \\
\leq & M(k, D, A) \int_{\Omega} \kappa(c)\left(\Phi_{e}^{\prime}\right)^{2} d x+\frac{(k+1) D}{2} \int_{\Omega} D c^{d-1 / 2-2 k-2}\left|c^{\prime}\right|^{2} d x
\end{aligned}
$$

by the assumption. Therefore

$$
\begin{aligned}
& \frac{\varepsilon_{e}}{k} \frac{d}{d t} \int_{\Omega} c^{-k} d x+(k+1) D \int_{\Omega} c^{-k-2}\left|c^{\prime}\right|^{2} d x \\
\leq & M(k, D, A) \int_{\Omega} \kappa(c)\left(\Phi_{e}^{\prime}\right)^{2} d x+\frac{(k+1) D}{2} \int_{\Omega} D c^{d-1 / 2-2 k-2}\left|c^{\prime}\right|^{2} d x .
\end{aligned}
$$

The conclusion follows from Lemma 6.1 by taking $k=d-1 / 2>0$.

Lemma 6.3. There exists $M>0$ such that

$$
\varepsilon_{e} \frac{d}{d t} \int_{\Omega} c^{2} d x+D \int_{\Omega}\left|c^{\prime}\right|^{2} d x \leq M \int_{\Omega} H(c) d x
$$

Proof. For $t \in(0, T)$, taking $\tilde{c}=c$ in (6.9) we obtain

$$
\frac{\varepsilon_{e}}{2} \frac{d}{d t} \int_{\Omega} c^{2} d x+D \int_{\Omega}\left|c^{\prime}\right|^{2} d x=\int_{\Omega} c S .
$$

Now taking $\Phi_{s}=c$ in (6.8) yields

$$
\int_{\Omega} c S=-\int_{\Omega} \sigma \Phi_{s}^{\prime} c^{\prime} \leq M(D, \sigma) \int_{\Omega} \sigma\left(\Phi_{s}^{\prime}\right)^{2} d x+\frac{D}{2} \int_{\Omega}\left|c^{\prime}\right|^{2} d x .
$$

The rest is the same as that of Lemma 6.2 and the proof is complete.

Remark. The way of using (6.9) in Lemmas 6.2 and 6.3 is readily justified if the inequalities in both lemmas are understood in the weak sense.

Proposition 6.4. Suppose $d=\alpha_{1} \alpha_{2} \leq 3 / 2$. Then for $t \in(0, T)$ a.e., there exists $M>0$ such that

$$
\int_{\Omega} H(c) d x \leq M e^{M t} .
$$



get

Proof. With the aid of Lemmas 6.2 and 6.3 , wee apply the Hölder inequality to

$$
\frac{d}{d t} \int_{\Omega}\left(c^{1 / 2-d}+c^{2}\right) d x \leq M \int_{\Omega} H(c) d x \leq M \int_{\Omega}\left(c^{1 / 2-d}+c^{2}\right) d x+M(|\Omega|),
$$

since by assumption $1 / 2+d \leq 2$. Now the conclusion follows from the Gronwall inequality.

Corollary 6.5. Suppose that the conditions in Lemma 6.2 and Proposition 6.4 hold. Then for $t \in(0, T)$ a.e., there exists $M>0$ such that

$$
\left\|\Phi_{s}\right\|_{L^{\infty}(\Omega)} \leq M e^{M t}
$$

Proof. By Lemma 6.1 and Proposition 6.4, we have

$$
\int_{\Omega}\left|\Phi_{s}^{\prime}\right|^{2} d x \leq M e^{M t}
$$

The conclusion follows immediately, with the aid of (6).

Using the above corollary, we can strengthen Lemma 6.1.

Lemma 6.6. For $k \geq \alpha_{2}$ and $t \in(0, T)$ a.e., there exists $M>0$ such that

$$
\begin{aligned}
& \int_{\Omega} \sigma\left(\Phi_{s}^{\prime}\right)^{2}\left(e^{-k \Phi_{s}}+e^{k \Phi_{s}}\right) d x+\int_{\Omega} \kappa(c)\left(\Phi_{e}^{\prime}\right)^{2}\left(e^{-k \Phi_{e}}+e^{k \Phi_{e}}\right) d x \\
& +\int_{\Omega}\left(c^{1 / 2-d} e^{\left(k+\alpha_{2}\right)\left(\Phi_{s}-\Phi_{e}\right)}+c^{1 / 2+d} e^{-\left(k+\alpha_{2}\right)\left(\Phi_{s}-\Phi_{e}\right)}\right) d x \\
\leq & M \int_{\Omega}\left(c^{1 / 2-k \alpha_{1}}+c^{1 / 2+k \alpha_{1}}\right) d x .
\end{aligned}
$$

Proof. The proof proceeds similarly as in Lemma 6.1. For $k>0$ and $t \in(0, T)$, use the test function $e^{k \Phi_{s}}$ in (6.8) and $e^{k \Phi_{e}}$ in (6.7) to obtain

$$
k \int_{\Omega} \sigma e^{k \Phi_{s}}\left(\Phi_{s}^{\prime}\right)^{2} d x+k \int_{\Omega} \kappa(c) e^{k \Phi_{e}}\left(\Phi_{e}^{\prime}\right)^{2} d x+\int_{\Omega}\left(e^{k \Phi_{s}}-e^{k \Phi_{e}}\right) S=0 .
$$

We shall estimate the last integral. Adopting the same notation as in Lemma 6.1, clearly we have

$$
\left|\int_{G_{3}}\left(e^{k \Phi_{s}}-e^{k \Phi_{e}}\right) S\right| \leq M\left(e^{k}\right) \int_{G_{3}}\left(c^{1 / 2-d}+c^{1 / 2+d}\right) \leq M \int_{\Omega} H(c),
$$

since both $\Phi_{s}$ and $\Phi_{e}$ are bounded on $G_{3}$ by the definition of $G_{3}$ and by Corollary 6.5. Next, we bound

$$
\begin{gathered}
\int_{G_{1}}\left(e^{k \Phi_{s}}-e^{k \Phi_{e}}\right) S= \\
=\int_{G_{1}} e^{k \Phi_{s}}\left(1-e^{-k\left(\Phi_{s}-\Phi_{e}\right)}\right)\left(c^{1 / 2-d} e^{\alpha_{2}\left(\Phi_{s}-\Phi_{e}\right)}-c^{1 / 2+d} e^{-\alpha_{2}\left(\Phi_{s}-\Phi_{e}\right)}\right) \\
\geq-\int_{G_{1}} e^{k \Phi_{s}}\left(1-e^{-k\left(\Phi_{s}-\Phi_{e}\right)}\right) c^{1 / 2+d} e^{-\alpha_{2}\left(\Phi_{s}-\Phi_{e}\right)} \\
\geq-L e^{-\alpha_{2}} \int_{G_{1}} c^{1 / 2+d}
\end{gathered}
$$


by Lemma 6.1, where $L=e^{k\left\|\Phi_{s}\right\|_{\infty}}$, since $0<1-e^{-k\left(\Phi_{s}-\Phi_{e}\right)}<1$.

Rewrite

$$
e^{k \Phi_{s}}-e^{k \Phi_{e}}=-l e^{-k\left(\Phi_{s}-\Phi_{e}\right)},
$$

where

$$
L \geq l=e^{k \Phi_{s}}\left(1-e^{k\left(\Phi_{s}-\Phi_{e}\right)}\right) \geq\left(1-e^{-k}\right) / L=L_{1}, \quad x \in G_{2} .
$$

It follows that

$$
\begin{aligned}
\int_{G_{2}}\left(e^{k \Phi_{s}}-e^{k \Phi_{e}}\right) S & =\int_{G_{2}} l\left(c^{1 / 2+d} e^{\left(-\left(k+\alpha_{2}\right)\left(\Phi_{s}-\Phi_{e}\right)\right.}-c^{1 / 2-d} e^{\left(\alpha_{2}-k\right)\left(\Phi_{s}-\Phi_{e}\right)}\right) \\
& \geq L_{1} \int_{G_{2}} c^{1 / 2+d} e^{-\left(k+\alpha_{2}\right)\left(\Phi_{s}-\Phi_{e}\right)}-L \int_{G_{2}} e^{\left(\alpha_{2}-k\right)\left(\Phi_{s}-\Phi_{e}\right)} c^{1 / 2-d}
\end{aligned}
$$

By Hölder's inequality, one has

$$
\begin{aligned}
& L \int_{G_{2}} e^{\left(\alpha_{2}-k\right)\left(\Phi_{s}-\Phi_{e}\right)} c^{1 / 2-d} \\
\leq & \frac{L_{1}}{2} \int_{G_{2}} c^{1 / 2+d} e^{-\left(k+\alpha_{2}\right)\left(\Phi_{s}-\Phi_{e}\right)}+M\left(k, \alpha_{2}, L_{1}, L\right) \int_{G_{2}} c^{1 / 2-k \alpha_{1}} .
\end{aligned}
$$

Therefore

$$
\int_{G_{2}}\left(e^{k \Phi_{s}}-e^{k \Phi_{e}}\right) S \geq \frac{L_{1}}{2} \int_{G_{2}} c^{1 / 2+d} e^{-\left(k+\alpha_{2}\right)\left(\Phi_{s}-\Phi_{e}\right)}-M \int_{G_{2}} c^{1 / 2-k \alpha_{1}} .
$$

Combining all above, we arrive at

$$
\begin{aligned}
& k \int_{\Omega} \sigma\left(\Phi_{s}^{\prime}\right)^{2} e^{k \Phi_{s}} d x+k \int_{\Omega} \kappa(c)\left(\Phi_{e}^{\prime}\right)^{2} e^{k \Phi_{e}} d x \\
& +\frac{L_{1}}{2} \int_{\Omega} c^{1 / 2+d} e^{-\left(k+\alpha_{2}\right)\left(\Phi_{s}-\Phi_{e}\right)} \leq M \int_{\Omega}\left(c^{1 / 2+d}+c^{1 / 2-k \alpha_{1}}\right),
\end{aligned}
$$

since

$$
\int_{G_{1} \cup G_{3}} c^{1 / 2+d} e^{-\left(k+\alpha_{2}\right)\left(\Phi_{s}-\Phi_{e}\right)} \leq e^{k+\alpha_{2}} \int_{G_{1} \cup G_{3}} c^{1 / 2+d} .
$$

Next, using $e^{-k \Phi_{s}}$ in (6.8) and $e^{-k \Phi_{e}}$ in (6.7), we obtain

$$
k \int_{\Omega} \sigma e^{-k \Phi_{s}}\left(\Phi_{s}^{\prime}\right)^{2} d x+k \int_{\Omega} \kappa(c) e^{-k \Phi_{e}}\left(\Phi_{e}^{\prime}\right)^{2} d x+\int_{\Omega}\left(e^{-k \Phi_{e}}-e^{-k \Phi_{s}}\right) S=0 .
$$

Proceeding similarly as before, we derive

$$
\int_{G_{2}}\left(e^{-k \Phi_{e}}-e^{-k \Phi_{s}}\right) S \geq-L \int_{G_{2}} c^{1 / 2-d} .
$$

and

$$
\int_{G_{1}}\left(e^{-k \Phi_{e}}-e^{-k \Phi_{s}}\right) S \geq \frac{L_{1}}{2} \int_{G_{1}} c^{1 / 2-d} e^{\left(k+\alpha_{2}\right)\left(\Phi_{s}-\Phi_{e}\right)}-M \int_{G_{1}} c^{1 / 2+k \alpha_{1}} .
$$


It follows that

$$
\begin{aligned}
& \left.k \int_{\Omega} \sigma \Phi_{s}^{\prime}\right)^{2} e^{-k \Phi_{s}} d x+k \int_{\Omega} \kappa(c)\left(\Phi_{e}^{\prime}\right)^{2} e^{-k \Phi_{e}} d x \\
& +\frac{L_{1}}{2} \int_{\Omega} c^{1 / 2-d} e^{\left(k+\alpha_{2}\right)\left(\Phi_{s}-\Phi_{e}\right)} \leq M \int_{\Omega}\left(c^{1 / 2-d}+c^{1 / 2+k \alpha_{1}}\right) .
\end{aligned}
$$

The proof is complete by combining (6.10) and (6.11).

We next estimate $c^{-1}$ in $L^{p}$-norm for all $p>0$.

Proposition 6.7. Suppose $d=\alpha_{1} \alpha_{2}>1$. Then for $p>0$ and $t \in(0, T)$ a.e., there exists $M=M(p)>0$ such that

$$
\int_{\Omega} c^{-p} d x \leq M
$$

Proof. For $t \in(0, T)$ and fixed $p>0$, multiply (6.9) by the test function $c^{-p-1}$ and integrate over $\Omega$ to obtain

$$
\frac{\varepsilon_{e}}{p} \frac{d}{d t} \int_{\Omega} c^{-p} d x+(p+1) D \int_{\Omega} c^{-p-2}\left|c^{\prime}\right|^{2} d x=-\int_{\Omega} c^{-p-1} S .
$$

We shall estimate the term on the right hand side as follows

$$
\begin{aligned}
& -\int_{\Omega} c^{-p-1} S \leq \int_{\Omega} c^{1 / 2+d-p-1} e^{-\alpha_{2}\left(\Phi_{s}-\Phi_{e}\right)} \\
\leq & \left(\int_{\Omega} c^{1 / 2+d} e^{-2 \alpha_{2}\left(\Phi_{s}-\Phi_{e}\right)}\right)^{1 / 2} \cdot\left(\int_{\Omega} c^{1 / 2+d-2(p+1)}\right)^{1 / 2} \\
\leq & \left(\int_{\Omega} c^{1 / 2-d}+\int_{\Omega} c^{1 / 2+d}\right)^{1 / 2} \cdot\left(\int_{\Omega} c^{1 / 2+d-2(p+1)}\right)^{1 / 2} \\
\leq & M\left(\int_{\Omega} c^{1 / 2-d}+\int_{\Omega} c^{1 / 2+d}\right) \cdot\left\|c^{2 d-2(p+1)}\right\|_{\infty}^{1 / 2} \\
\leq & M(T)\left\|c^{d-(p+1)}\right\|_{\infty}=M(T)\left\|c^{-p / 2}\right\|_{\infty}^{2(p+1-d) / p}
\end{aligned}
$$

by Lemma 6.6 with the choice $k=\alpha_{2}$. On the other hand, the quantity $\min _{x \in \Omega} c^{-p / 2}$ is uniformly bounded for all $t \in\left[0, T\right.$ ) (depending only on the initial data $c_{0}$ and of course $p$ ) since the conservation of $c$

$$
\int_{\Omega} c=\text { const. }
$$

It follows that

$$
\left\|c^{-p / 2}\right\|_{\infty} \leq M p\left(\int_{\Omega} c^{-p-2}\left|c^{\prime}\right|^{2} d x\right)^{1 / 2}+M
$$

Therefore,

$$
-\int_{\Omega} c^{-p-1} S \leq M\left(\int_{\Omega} c^{-p-2}\left|c^{\prime}\right|^{2} d x\right)^{d_{1}}+M
$$

where

$$
d_{1}=\frac{1-d}{p}+1<1
$$


by the assumption $d=\alpha_{1} \alpha_{2}>1$. Combining the above estimates yields

$$
-\int_{\Omega} c^{-p-1} S \leq M+(p+1) D\left(\int_{\Omega} c^{-p-2}\left|c^{\prime}\right|^{2} d x\right),
$$

since $d_{1}<1$. We finally reach

$$
\frac{\varepsilon}{p} \frac{d}{d t} \int_{\Omega} c^{-p} d x \leq M
$$

and the conclusion follows.

Corollary 6.8. Suppose that the conditions of Lemma 6.2 and Propositions 6.4 and 6.7 hold and that there exists $k>0$ such that

$$
\kappa(c) \geq c^{k}, \quad c<<1 .
$$

Then for $t \in(0, T)$ a.e., there exists $M>0$ such that

$$
\left\|\Phi_{e}\right\|_{\infty} \leq M e^{M t}
$$

Proof. By the assumption, Lemma 6.1 and Propositions 6.4 and 6.7 hold. We have

$$
\begin{aligned}
& \int_{\Omega}\left|\Phi_{e}^{\prime}\right| d x \leq\left(\int_{\Omega} \kappa(c)\left|\Phi_{e}^{\prime}\right|^{2} d x\right)^{1 / 2}\left(\int_{\Omega} \kappa^{-1}(c) d x\right)^{1 / 2} \\
& \leq M\left(\int_{\Omega} c^{-k} d x\right)^{1 / 2} \leq M .
\end{aligned}
$$

Using Lemma 6.6 with $k=\alpha_{2}$, Propositions 6.4 and 6.7 and Corollary 6.5, we infer that for all $t \in(0, T)$ a.e.,

$$
\begin{aligned}
\int_{\Omega} e^{\alpha_{2} \Phi_{e}} & =\int_{\Omega} c^{1 / 2-d} e^{\alpha_{2} \Phi_{e}} c^{d-1 / 2} \\
& \leq\left(\int_{\Omega} c^{1 / 2-d} e^{2 \alpha_{2} \Phi_{e}}\right)^{1 / 2} \cdot\left(\int_{\Omega} c^{d-1 / 2}\right)^{1 / 2} \leq M
\end{aligned}
$$

since $d-1 / 2 \leq 2$. Similarly

$$
\int_{\Omega} e^{-\alpha_{2} \Phi_{e}} d x \leq M
$$

The conclusion follows.

6.2. Main result of global existence. In this section, we shall establish a global existence of a weak solution to the system (6.2-6.5). First, we derive an a priori estimate below, using the results obtained in the previous subsection.

We need the following supremum estimate.

Lemma 6.9. Let $\Omega \in \mathbf{R}^{n}$ be bounded and smooth. Suppose that $u$ is a nonnegative subsolution of the initial-boundary value problem

$$
\frac{\partial(E u)}{\partial t}-\frac{\partial}{\partial x}\left(D \frac{\partial u}{\partial x}\right)=K(u(x)+1), \quad(x, t) \in \Omega \times(0, T),
$$


where $E$ and $D$ are positive piece-wise constants, with homogeneous Neumann boundary conditions:

$$
\frac{\partial u}{\partial n}=0 \text { for } x \in \partial \Omega
$$

and also the initial condition:

$$
u(x, 0)=u_{0}(x), \quad x \in \Omega
$$

with $u_{0} \in L^{\infty}$. Then there exists $M>0$ such that

$$
\sup _{\Omega} u(\cdot, t) \leq \sup _{\Omega} u_{0}(x)+M
$$

Proof. The proof is standard via boot-strap, see for example [10] or $[15]^{2}$. $\mathrm{Z}$

Now we can prove the following a priori estimate.

Theorem 6.10. Let $d=\alpha_{1} \alpha_{2} \in(1,3 / 2]$, Suppose that there exists $k \geq 3 / 2+d$, $k_{1}>0$ such that

$$
c^{k} \leq \kappa(c) \leq k_{1} c^{3 / 2+d}, \quad c \leq 1 .
$$

Then there exists $M>0$ such that

$$
\left\|\Phi_{s}\right\|_{L^{\infty}(\Omega)} \leq M e^{M t}, \quad\left\|\Phi_{e}\right\|_{L^{\infty}(\Omega)} \leq M e^{M t}
$$

and

$$
\frac{1}{M} \leq c(x, t) \leq M, \quad(x, t) \in \Omega \times(0, T)
$$

Proof. By Corollaries 6.5 and 6.8, we have (6.12). We shall prove the first inequality in (6.13), the second being essentially the same. Put

$$
u(x, t)=c^{-1}(x, t) .
$$

One readily verifies that $u$ satisfies the equation

$$
\begin{aligned}
\frac{\partial\left(\varepsilon_{e} u\right)}{\partial t}-\frac{\partial}{\partial x}\left(D \frac{\partial u}{\partial x}\right) & = \\
-c^{-2}\left(\frac{\partial\left(\varepsilon_{e} c\right)}{\partial t}-\frac{\partial}{\partial x}\left(D \frac{\partial c}{\partial x}\right)\right)-2 D c^{-3} c^{\prime 2} & =-c^{-2} S-2 D c^{-3} c^{\prime 2}
\end{aligned}
$$

with the initial-Neumann boundary condition

$$
u(x, 0)=1 / c_{0}(x), \quad \frac{\partial u}{\partial n}=0 \quad \text { at } x=0 \text { and } L .
$$

In particular, $u$ is a positive subsolution of the equation

$$
\frac{\partial\left(\varepsilon_{e} u\right)}{\partial t}-\frac{\partial}{\partial x}\left(D \frac{\partial u}{\partial x}\right)=M(u+1), \quad(x, t) \in \Omega \times(0, T),
$$

\footnotetext{
${ }^{2}$ Again, Initial-Boundary (homogeneous Dirichlet) value problems were treated, see footnote 1
} 
since

$$
\left|e^{-\alpha_{2}\left(\Phi_{s}-\Phi_{e}\right)}\right| \leq M
$$

by $(6.12)$ and

$$
\begin{aligned}
-c^{-2} S-2 D c^{-3} c^{\prime 2}= & c^{1 / 2+d-2} e^{-\alpha_{2}\left(\Phi_{s}-\Phi_{e}\right)} \\
& -c^{1 / 2-d-2} e^{\alpha_{2}\left(\Phi_{s}-\Phi_{e}\right)}-2 D c^{-3} c^{\prime 2} \\
\leq & M c^{d-3 / 2}=M u^{3 / 2-d} \leq M(u+1) .
\end{aligned}
$$

Now (6.13) follows from Lemma 6.9 immediately and the theorem is proved.

Local in time existence and uniqueness can be proved in the similar way as Theorem 4.1. With the aid of Theorem 6.10, the following global existence result is standard via a continuation argument.

Theorem 6.11. Assume further that

$$
d=\alpha_{1} \alpha_{2} \in(1,3 / 2],
$$

and that there exists $k \geq 3 / 2+d, k_{1}>0$ such that

$$
c^{k} \leq \kappa(c) \leq k_{1} c^{3 / 2+d}, \quad c \leq 1 .
$$

Then exists a solution to the system of (6.1-6.3) for all $T>0$.

\section{REFERENCES}

[1] R.A. Adams, Sobolev space, New York, Academic press, 1975.

[2] Y. Chen And J.W. Evans, Thermal analysis of lithium-ion batteries, J. Electrochem. Soc., 143, 2708 (1996).

[3] M.Doyle, J. Newman, A.S. Gozdz, C.N. Schmutz, and J.-M. Tarascon, Comparison of modeling predictions with experimental data from plastic lithium ion cells, J. Electrochem. Soc., 143, 1890 (1996).

[4] A. Friedman, Mathematics in Industrial Problems, Part 7, 229-240 (1995).

[5] T.F. Fuller, M. Doyle And J. Newman, Simulation and optimization of the dual lithium ion insertion cell, J. Electrochem. Soc., 141, 1 (1994).

[6] M. Giaquinta, Multiple integrals in the calculus of variations and nonlinear elliptic systems, Annals of mathematics studies, no. 105, Princeton University Press, 1983.

[7] D. Gilbarg And N.S. Trudinger, Elliptic Partial Differential equations of Second Order, Springer-Verlag, Heidelberg, New York, 1977.

[8] W.B. Gu And C.Y. WAng, Thermal and Electrochemical Coupled Modeling of a Lithium-Ion Cell, R.A. Marsh, Z. Oguma, J. Prakash, and S. Surampudi, Editors, The Electrochemical Society Series, PV 99-29, 748 (1999).

[9] J.-S. Hong, H. Maleki, S. Al Hallaj, L. Redey and J.R. Selman, ElectrochemicalCalorimetric studies of lithium-ion cells, J. Eletrochem. Soc., 145, 1489 (1998).

[10] O.A. Ladyzenskaja, V.A. Solonnikov and N.N. Ural'tzeva, Linear and quasilinear equations of parabolic type, Trans. Math. Mono., AMS, Vol. 23 (1968).

[11] O.A. Ladyzenskaya and N.N. Ural'tseva, Linear and quasilinear elliptic equations, English Transl., Academic Press, New York, 1968.

[12] J.S. Newman, Electrochemical Systems, Second Edition, 1991.

[13] J.S. Newman, The Fundamental Principles of Current Distribution and Mass Transport in Electrochemical Cells, Allen J. Bard, Ed., Electro-analytical Chemistry, 6 (1973), 187.

[14] J. Moser, A new proof of de Giorgi's theorem concerning the regularity problem for elliptic differential equations, Comm. Pure Appl. Math., 13 (1960), pp. 457-468.

[15] J. Moser, A Harnack inequality for parabolic differential equations, Comm. Pure Appl. Math., 17 (1964), pp. 101-134.

[16] C.Y. WANG, W.B. Gu AND B.Y. Liaw, Micro-macroscopic coupled modeling of batteries and fuel cells I. Model development, J. Eletrochem. Soc., 145, 3407 (1998). 
